\title{
Acute Posterior Cranial Fossa Hemorrhage-Is Surgical Decompression Better than Expectant Medical Management?
}

\author{
M. S. Luney ${ }^{1}$ S. W. English ${ }^{2,3} \cdot$ A. Longworth ${ }^{3} \cdot$ J. Simpson $^{3} \cdot$ S. Gudibande ${ }^{4}$. \\ B. Matta ${ }^{3} \cdot$ R. M. Burnstein ${ }^{3} \cdot$ T. Veenith ${ }^{3,4}$
}

Published online: 12 April 2016

(c) The Author(s) 2016. This article is published with open access at Springerlink.com

\begin{abstract}
Background To compare the in-hospital mortality and institutional morbidity from medical therapy (MT), external ventricular drainage (EVD) and suboccipital decompressive craniectomy (SDC) following an acute hemorrhagic posterior cranial fossa stroke (PCFH) in patients admitted to the neurosciences critical care unit (NCCU). Retrospective observational single-center cohort study in a tertiary care center. All consecutive patients $(n=104)$ admitted with PCFH from January 1st 2005-December 31st 2011 were included in the study.

Methods All patients with a PCFH were identified and confirmed by reviewing computed tomography of the brain reported by a specialist neuroradiologist. Management decisions (MT, EVD, and SDC) were identified from operative notes and electronic patient records.

Results Following a PCFH, $47.8 \%(n=11)$ patients died after EVD placement without decompression, $45.7 \%$ $(n=16)$ died following MT alone, and $17.4 \%(n=8)$ died following SDC. SDC was associated with lower mortality compared to MT with or without $\operatorname{EVD}\left(\chi^{2}\right.$ test $p=0.006, p=0.008)$. Age, ICNARC score, brain stem
\end{abstract}

M. S. Luney and S. W. English have contributed equally to this study.

T. Veenith

tv227@cam.ac.uk

1 School of Clinical Medicine, Addenbrooke's Hospital, Cambridge, UK

2 Department of Medicine (Critical Care), The Ottawa Hospital, Ottawa, Canada

3 Neurosciences Critical Care Unit, Addenbrooke's Hospital, Cambridge, UK

4 Critical Care Unit, University Hospital Birmingham NHS Foundation Trust, Edgbaston, UK involvement, and hematoma volume did not differ significantly between the groups. There was a statistically significant increase in hydrocephalus and intraventricular bleeds in patients treated with EVD placement and SDC ( $\chi^{2}$ test $p=0.02$ ). Median admission Glasgow Coma Scale scores for the MT only, MT with EVD, and SDC groups were 8,6 , and 7 , respectively (ranges $3-15,3-11$ and 3-13) and did not differ significantly (Friedman test: $p=0.89$ ). SDC resulted in a longer NCCU stay (mean of 17.4 days, standard deviation $=15.4, p<0.001)$ and increased incidence of tracheostomy (50 vs. $17.2 \%, p=0.0004$ ) compared to MT with or without EVD.

Conclusions SDC following PCFH was associated with a reduction in mortality compared to expectant MT with or without EVD insertion. A high-quality multicenter randomized control trial is required to evaluate the superiority of SDC for PCFH.

Keywords Infratentorial hemorrhage .

Cerebellar hemorrhage $\cdot$ Critical care .

External ventricular drain .

Suboccipital decompressive craniectomy - Intensive care

Tracheostomy

\section{Introduction}

The posterior cranial fossa (PCF) is the deepest and most confined space in the skull. It has a limited ability to accommodate an expansion of its contents. PCF hemorrhage (PCFH) accounts for between 9 and $15 \%$ of all intracranial hemorrhage in Europe and North America [1-3]. When left to its natural course, it can result in life-threatening complications such as compression of the pons and medulla, resulting in impaired consciousness, 
respiratory failure, and lower cranial nerve dysfunction. Acute hydrocephalus may also result due to the obstruction of the fourth ventricle leading to herniation of the PCF contents.

The reported mortality from PCFH varies widely and can range from 20 to $75 \%$, [2, 4-9] depending on the center. The survivors have significant morbidity requiring longterm institutional care. Although management in neurocritical care units have reduced these complications, there is a paucity of evidence to support the current guidelines to manage PCFH [3, 10-13]. Surgical interventions such as suboccipital decompression (SDC) of the PCF have been shown to reduce mortality in well-conducted prospective studies [2]. Bilateral SDC is documented to be safe in patients with malignant cerebellar edema following PCFH, and long-term outcomes following surgery were acceptable [14]. Despite various clinical guidelines, there is institutional variability in the management of PCFH [15].

The aim of our study is to investigate the epidemiology, institutional mortality, and morbidity in patients with PCFH managed in a tertiary center neurosciences critical care unit (NCCU).

\section{Materials and Methods}

\section{Setting}

All patients admitted to the Neurosciences' Critical Care Unit (NCCU) at Cambridge University Hospitals NHS Foundation Trust with a PCFH from January 1st 2005 to December 31st 2011 were included in this study. This study was reviewed by Cambridge East Research Ethics Committee, and a formal review by ethics committee was waived.

\section{Patient Identification}

Addenbrooke's Hospital NCCU is a 21-bed intensive care unit specializing in the care of patients with neurological disorders. Patients are managed by dedicated neurocritical care physicians, nurses, and allied health care professionals. All NCCU admissions are prospectively logged in the intensive care national audit and research centre (ICNARC) database which includes primary diagnosis, demographic, and clinical data. This high-quality national database is maintained by a multidisciplinary team which includes research nurses and database administrators. The quality of the accrued data is routinely checked and verified by independent audits to compare our institutional performance with other intensive care units in the United Kingdom.

All patients admitted between January 1st 2005-December 31st 2012 with a primary neurosurgical or neurology admitting team were considered for inclusion. Patients admitted with a non-PCF stroke were excluded; in remaining patients, the brain computed tomogram (CT) and subsequent report by a neuroradiologist were reviewed. All patients with the evidence of a PCFH on CT scan were included in this study. In patients where the diagnosis was unclear, neuroimaging was reviewed by a second (SWE) and a third reviewer (TV) to reach a consensus. Patients with a primary subarachnoid hemorrhage, trauma, or malignancy were excluded from this study.

Descriptive data such as patient demographics and baseline Glasgow Coma Scale (GCS) score were retrieved from the ICNARC database. Electronic discharge, operative notes, and death summaries when available were also reviewed. Primary and secondary outcome data were obtained from the information collected on the ICNARC database.

\section{Outcomes}

Our objective was to compare medical management (MT) with or without external ventricular drain (EVD) to SDC. Our primary outcome measures of interest were NCCU and in-hospital mortality. Morbidity was assessed using NCCU and hospital length of stay as well as the incidence of tracheostomy.

\section{Statistical Analysis}

Statistical analyses were conducted using Statview (Version 5, 1998, SAS Institute Inc., Cary, North Carolina, USA). Paired non-parametric data were compared using the Mann-Whitney $U$ test, and non-parametric data with three or more datasets were compared using the Freidman test. Appropriate Bonferroni corrections were applied, and a $p$ value of $<0.05$ was considered statistically significant.

\section{Results}

\section{Patient Characteristics}

Between January 1st 2005 and December 31st 2011, 5915 patients were admitted to the NCCU. Once our exclusion criteria were applied, we identified 104 patients with PCFH. Emergency admissions to Addenbrooke's Hospital accounted for $48.1 \%$ of PCFH patients $(n=50)$. The remaining $51.9 \%(n=54)$ were secondary referrals from other hospitals.

The demographic and baseline clinical characteristics of patients with PCFH are summarized in Table 1. Admission GCS of MT patients with or without EVD did not differ significantly $(p=0.11)$. GCS did not differ in patients managed surgically compared to MT with $(p=0.40)$ or without $\operatorname{EVD}(p=0.25)$. The GCS could not be 
Table 1 Patient characteristics

\begin{tabular}{|c|c|c|c|c|c|}
\hline & Overall & MT & EVD & SDC & $p$ value \\
\hline Number of patients (\%) & $104(100)$ & $35(34)$ & $23(22)$ & $46(44)$ & - \\
\hline Male $(\%)$ & $64(62)$ & $25(71)$ & $14(61)$ & $25(54)$ & - \\
\hline Age in years $( \pm \mathrm{SD})$ & $57.7 \pm 15.9$ & $56.1 \pm 17.4$ & $57.6 \pm 17.7$ & $58.8 \pm 13.9$ & 0.88 \\
\hline GCS score (range) & $7(3-15)$ & $8(3-15)$ & $6(3-11)$ & $7(3-13)$ & 0.89 \\
\hline ICNARC score $( \pm \mathrm{SD})$ & $18.7 \pm 6.8$ & $19.2 \pm 8.1$ & $18.7 \pm 8.0$ & $18.4 \pm 5.1$ & 0.37 \\
\hline Volume of bleed $(\mathrm{mls})( \pm \mathrm{SD})$ & $8.2 \pm 8.8$ & $7.1 \pm 8.2$ & $7.0 \pm 7.6$ & $12 \pm 8$ & 0.45 \\
\hline Presence of hydrocephalus $(\%)$ & 70.0 & 48.5 & 75 & 90.3 & $0.02 *$ \\
\hline Presence of IVH (\%) & 57.0 & 36.6 & 85.7 & 70.5 & $0.001 *$ \\
\hline Brain stem involvement $(\%)$ & 16.1 & 15.6 & 13.3 & 19 & 0.13 \\
\hline
\end{tabular}

The demographic and clinical characteristics of patients with spontaneous posterior cranial fossa hemorrhage split by management strategy. GCS is defined as the lowest Glasgow coma scale score within first $24 \mathrm{~h}$ of admission

$M T$ medical therapy without external ventricular drainage; EVD medical therapy with EVD; $S D C$ surgical decompressive craniotomy; $S D$ standard deviation; IVH intraventricular hemorrhage

* Statistically significant

ascertained for 28 patients $(26.9 \%)$ due to sedation upon NCCU admission. Age, ICNARC score, and brain stem involvement did not differ significantly between groups. There was no significant progression in initial CT hematoma volume compared to $24 \mathrm{~h}$ post admission. At $24 \mathrm{~h}$, the mean volume in milliliters for MT was 7.1, $7 \mathrm{mls}$ for MT with EVD, and SDC $12 \mathrm{mls}(p=0.45)$. However, there was a statistically significant presence of hydrocephalus and intraventricular hemorrhage in patients treated with EVD and SDC $(p<0.05)$.

\section{Primary Outcome}

Of the 104 patients studied, $69(66.3 \%)$ survived their admission from the NCCU to discharge home or to a rehabilitation unit. Thirty one patients died in NCCU and a further four following discharge from NCCU. Survival to discharge or death in the hospital is summarized in Table 2. Mortality was the lowest in the surgically treated patients and those receiving medical therapy with EVD insertion had higher mortality compared to surgical decompression (47.8 vs. $17.4 \%, p=0.008, \mathrm{OR}=4.4$, $\mathrm{RR}=2.8$ ) and medical therapy without EVD (45.7 vs. $17.4 \%, p=0.006, \mathrm{OR}=4.0, \mathrm{RR}=2.6)$. In surgically managed patients, the mean time to surgical decompression was less than $24 \mathrm{~h}$.

\section{Patient Characteristics Based on Mortality}

Admission GCS and ICNARC scores differed significantly between PCFH survivors and non- survivors (see Table 3). In the subgroup of patients with an admission GCS less than 8 , a higher proportion of surgically managed patients survived $(73.7 \%)$ compared to MT patients $(26.9 \%)$ This was inclusive of patients treated with an EVD. However, there was no significant difference in mean GCS $(p=0.40)$, ICNARC score $(p=0.44)$, and age $(p=0.44)$. Baseline GCS was not significantly different in MT patients without EVD compared to patients with an $\operatorname{EVD}(p=0.91)$.

\section{Secondary Outcomes}

An EVD was inserted in $93.5 \%(n=43)$ of patients undergoing decompression compared to $39.7 \%(n=23)$ of patients managed non-surgically. Surgically managed patients had a longer NCCU stay compared to those with MT, including patients with an EVD (17.4 \pm 15.4 versus $7.2 \pm 8.8$ (mean $\pm \mathrm{SD}$ ) respectively, $p<0.001,95 \% \mathrm{CI}$ $-14.9,-5.4)$. Hospital length of stay was longer in surgically managed patients $(42.2 \pm 53.5$ versus. $21.7 \pm 28.0$ (mean $\pm \mathrm{SD}), p=0.013,95 \% \mathrm{CI}-36.6,-4.3$ ) (Table 4). MT managed patients surviving PCFH experienced similar lengths of stay in hospital, regardless of EVD insertion $(p=0.32)$. Surgically managed patients had a longer

Table 2 Hospital survival

\begin{tabular}{llllr}
\hline & Overall & MT & EVD & SDC \\
\hline Alive $(\%)$ & $69(66.3)$ & $19(54.3)$ & $12(52.2)$ & $38(82.6)$ \\
Dead (\%) & $35(33.7)$ & $16(45.7)$ & $11(47.8)$ & $8(17.4)$ \\
\hline
\end{tabular}

The proportion of patients surviving to discharge following PCFH with admission to NCCU stratified by management strategy $M T$ medical therapy only; $E V D$ external ventricular drain, $S D C$ suboccipital decompressive craniectomy; $S D$ standard deviation 
Table 3 Patient characteristics based on mortality

\begin{tabular}{|c|c|c|c|c|}
\hline & Overall & Alive & Dead & $p$ value \\
\hline Number of patients (\%) & $104(100)$ & $69(66.3)$ & $35(33.7)$ & - \\
\hline Male $(\%)$ & $64(62)$ & $38(55)$ & $26(74)$ & - \\
\hline Age in years $( \pm$ SD $)$ & $57.7 \pm 15.9$ & $56.1 \pm 15.9$ & $60.8 \pm 15.7$ & 0.15 \\
\hline GCS score (range) & $7(3-15)$ & $8(3-15)$ & $4(3-9)$ & $<0.0001$ \\
\hline ICNARC score $( \pm S D)$ & $18.7 \pm 6.8$ & $16.7 \pm 5.9$ & $22.8 \pm 6.6$ & $<0.0001$ \\
\hline
\end{tabular}

Demographics and clinical characteristics of patients with acute non-traumatic PCF hemorrhage based on primary outcome

Table 4 Secondary outcomes from PCF hemorrhage

\begin{tabular}{|c|c|c|c|c|}
\hline & Overall & MT & EVD & SDC \\
\hline \multicolumn{5}{|l|}{ Mean length of stay $(d)( \pm S D)$} \\
\hline NCCU, all & $11.7 \pm 13.1$ & $6.0 \pm 7.7$ & $9.1 \pm 10.3$ & $17.4 \pm 15.4$ \\
\hline NCCU, survivors & $15.2 \pm 14.0$ & $8.0 \pm 7.9$ & $12.3 \pm 12.2$ & $20.3 \pm 15.4$ \\
\hline NCCU, non-survivors & $3.7 \pm 5.1$ & $2.6 \pm 6.2$ & $5.0 \pm 5.4$ & $3.7 \pm 2.6$ \\
\hline Hospital, all & $30.8 \pm 42.2$ & $21.6 \pm 30.3$ & $22.0 \pm 24.7$ & $42.2 \pm 53.5$ \\
\hline Hospital, survivors & $41.3 \pm 46.2$ & $26.4 \pm 30.1$ & $37.0 \pm 26.1$ & $50.1 \pm 55.5$ \\
\hline Hospital, non-survivors & $10.1 \pm 21.3$ & $15.9 \pm 30.6$ & $5.5 \pm 4.9$ & $4.8 \pm 3.7$ \\
\hline Tracheostomy: number of patients, $(\%)$ & $33(31.7)$ & $4(11.4)$ & $6(26.1)$ & $23(50)$ \\
\hline
\end{tabular}

$M T$ medical therapy only; $E V D$ external ventricular drain; $S D C$ suboccipital decompressive craniectomy; $d$ days; $S D$ standard deviation; $N C C U$ neurosciences critical care unit

hospital stay compared to those medically managed with or without an EVD. However, this did not reach statistical significance $(p=0.088)$, despite inclusion of those with an EVD ( $p=0.794)$. Surgical decompression (Fig. 1) resulted in a higher incidence of tracheostomy compared to MT with or without an EVD (50 vs. $17.2 \%, p=0.0004$ ).

\section{Discussion}

$47.1 \%$ of patients admitted to a tertiary center NCCU following PCFH underwent SDC.

SDC was associated with an increased survival in patients compared to MT with or without EVD insertion. Surgery was offered more frequently for patients with hydrocephalus and intraventricular hemorrhage. Baseline GCS, ICNARC scores, and brainstem involvement were similar in all treatment groups. In our study, the use of EVD without surgical decompression compared to medical management alone was associated with an increase in mortality.

SDC is a treatment option for the management of deteriorating patients with acute $\mathrm{PCFH}$ and rising PCF pressure [11]. Previous small studies have shown an association of SDC for PCFH and reduced mortality and morbidity, despite low initial GCS $[12,13]$. Although recommended in clinical guidelines, evidence is sparse to recommend SDC routinely $[2,16]$. Management strategies proposed by current guidelines are mixed $[3,11,17,18]$. The guidelines for the management of spontaneous intracerebral hemorrhage produced by the American Heart Association Stroke Council [11] recommends surgical removal of hematoma for patients with PCFH and neurologic deterioration, brainstem compression, or hydrocephalus. This guidance has been developed largely from observational and retrospective studies, some of which supports EVD as a viable independent treatment option [19]. These interventions are dependent on institutional guidelines focusing on the degree of $4^{\text {th }}$ ventricle occlusion [20] or hematoma dimensions [2]. A strategy focussed solely on EVD may be ineffective compared to SDC in the management of PCF hemorrhage.

Our data support the use of SDC rather than expectant medical management with or without EVD. The American Stroke Association recommendations favoring SDC were made in 2015 [11] and the management choices may have changed following these recommendations. In this study, a low GCS and ICNARC (a measure of peri-admission morbidity) score were associated with increased mortality. Previous studies are in agreement with our institutional data regarding the importance of GCS as a prognostic marker after PCFH $[13,21]$. Our study has demonstrated a trend toward increased survival following SDC, even in patients presenting with low GCS.

Infratentorial lesions may affect lower cranial nerve function leading to tracheostomy. This may be used to demonstrate institutional morbidity and the severity of stroke $[22,23]$. Despite improved mortality, the incidence 


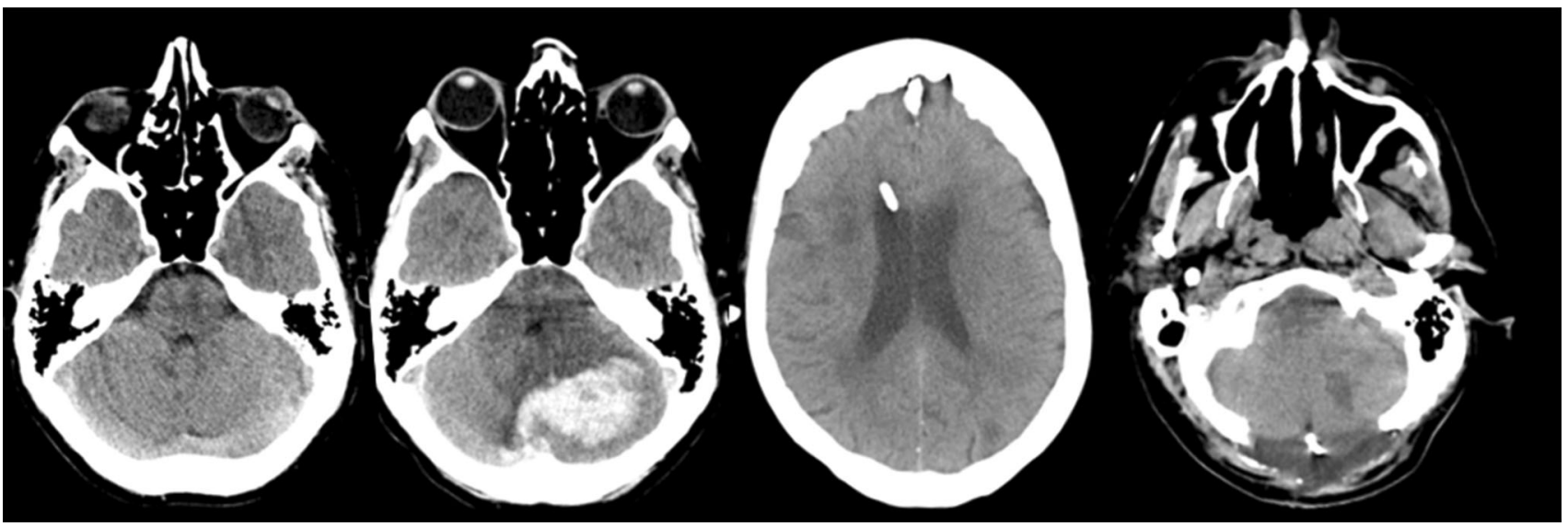

Fig. 1 Computed tomography showing the head of a patient admitted with a posterior cranial fossa bleed. This patient was initially treated with external ventricular drain followed by posterior cranial fossa decompression

of tracheostomy in surgically managed patients was higher than medically managed patients $(50.0$ vs. $17.1 \%$, Table 3 ). This may be explained by the added edema, injury, and the time required for surgical recovery following decompression of the posterior cranial fossa.

In this study, patients who died had a shorter duration of ICU and hospital stay compared to survivors. This may be related to the withdrawal of care as a response to severe brain injury. This trend is also seen in other patient cohorts, for example, in traumatic brain injury [20]. Longer ICU stay in surviving surgical patients was likely due to surgical recovery time and prolonged weaning from mechanical ventilation [24]. A recent study suggests that these patients may benefit from early tracheostomy to facilitate weaning from mechanical ventilation [25].

One of the strengths of our study is the completeness of data over a period of 7 years and the use of a comprehensive and validated database. Previously described literature has been based on smaller cohorts or a less rigorous case definition [2, 6, 8, 12, 13, 26-28], making this one of the largest studies of its kind. Limitations of our study include the retrospective and non-randomized nature of data used. There is potential for selection bias as our unit is a specialist center within a tertiary care hospital and as such attracts the "unwell" patients requiring intensive care treatment. Futility and patient outcome may have biased the transfer of patients to our tertiary referral center thus skewing results. However, in our study, $51.2 \%$ of patients with $\mathrm{PCFH}$ were referrals from other hospitals. In our region, Glasgow coma score was used as a tool to trigger referral in patients with PCFH. In this study, median GCS was 5.5 for patients presenting directly to emergency department and those referred for tertiary management.

There are many other factors not included in our study that could play a role in the management decision to perform or withhold surgical decompression. They include trends in GCS score, cerebral perfusion, pupillary responses, the degree of $4^{\text {th }}$ ventricle collapse, and the presence of cerebral edema. These highlight the need for a carefully planned study incorporating the above factors, regarding the management of PCFH.

In the absence of a volumetric magnetic resonance imaging for our patients, a CT brain was used to measure the volume of bleed. This approach could introduce errors due to partial volume effects and beam-hardening artifacts. The results of our study cannot be generalized to other posterior fossa pathologies including traumatic and spontaneous subarachnoid hemorrhage [20].

Our study only addresses ICU and in-hospital mortality and morbidity and not long-term functional and neurocognitive outcomes. Although our findings suggest that an aggressive surgical approach may be associated with a reduced mortality after $\mathrm{PCFH}$, it remains unclear which patients are best suited to this approach and whether functional morbidity and long-term mortality outcomes are affected as suggested in other centers [8, 24]. Our results clearly demonstrate the requirement for a high-quality multicenter randomized trial (RCT) in patients with PCFH.

\section{Conclusions}

Suboccipital decompression was only undertaken in $47 \%$ of patients with an acute hemorrhage in the posterior cranial fossa following admission to a neurocritical care unit. SDC was associated with a favorable survival compared to medical management alone (with or without EVD insertion) in our tertiary referral hospital. Definitive conclusions cannot be drawn from these data and a high-quality randomized control trial should be performed in this cohort of patients. 
Open Access This article is distributed under the terms of the Creative Commons Attribution 4.0 International License (http:// creativecommons.org/licenses/by/4.0/), which permits unrestricted use, distribution, and reproduction in any medium, provided you give appropriate credit to the original author(s) and the source, provide a link to the Creative Commons license, and indicate if changes were made.

\section{References}

1. Béjot Y, Cordonnier C, Durier J, Aboa-Eboulé C, Rouaud O, Giroud M. Intracerebral haemorrhage profiles are changing: results from the Dijon population-based study. Brain. 2013; doi:10.1093/brain/aws349.

2. Kirollos RW, Tyagi AK, Ross SA, van Hille PT, Marks PV. Management of spontaneous cerebellar hematomas: a prospective treatment protocol. Neurosurgery. 2001;49(6):1378-86 discussion 1386

3. Amar AP. Controversies in the neurosurgical management of cerebellar hemorrhage and infarction. Neurosurg Focus. 2012;32(4):E1.

4. Ott KH, Kase CS, Ojemann RG, et al. Cerebellar hemorrhage: diagnosis and treatment. A review of 56 cases. Arch Neurol (Chicago). 1974;31:160-7.

5. Donauer E, Loew F, Faubert C, Alesch F, Schaan M. Prognostic factors in the treatment of cerebellar haemorrhage. Acta Neurochir (Wien). 1994;131:59-66.

6. van Loon J, Van Calenbergh F, Goffin J, Plets C. Controversies in the management of spontaneous cerebellar haemorrhage. A consecutive series of 49 cases and review of the literature. Acta Neurochir (Wien). 1993;122:187-93.

7. Kobayashi S, Sato A, Kageyama Y, Nakamura H, Watanabe Y, Yamaura A. Treatment of hypertensive cerebellar hemorrhagesurgical or conservative management? Neurosurgery. 1994;34:246-51.

8. Sacco S, Marini C, Toni D, Olivieri L, Carolei A. Incidence and 10 -year survival of intracerebral hemorrhage in a populationbased registry. Stroke. 2009;40(2):394-9.

9. Dammann P, Asgari S, Bassiouni H, Gasser T, Panagiotopoulos V, Gizewski ER, et al. Spontaneous cerebellar hemorrhageexperience with 57 surgically treated patients and review of the literature. Neurosurg Rev. 2011;34:77-86.

10. Da Pian R, Bazzan A, Pasqualin A. Surgical versus medical treatment of spontaneous posterior fossa haematomas: a cooperative study on 205 cases. Neurol Res. 1984;6(3):145-51.

11. Hemphill J Claude, Greenberg Steven M, Anderson Craig S, Becker Kyra, Bendok Bernard R, Cushman Mary, Fung Gordon $\mathrm{L}$, et al. Guidelines for the management of spontaneous intracerebral hemorrhage: a guideline for healthcare professionals from the American Heart Association/American Stroke Association. Stroke. 2015;. doi:10.1161/STR.0000000000000069.

12. Mathew P, Teasdale G, Bannan A, Oluoch-Olunya D. Neurosurgical management of cerebellar haematoma and infarct. J Neurol Neurosurg Psychiatry. 1995;59(3):287-92.

13. Yanaka K, Meguro K, Fujita K, Narushima K, Nose T. Immediate surgery reduces mortality in deeply comatose patients with spontaneous cerebellar hemorrhage. Neurol Med Chir (Tokyo). 2000;40(6):295-9 discussion 299.
14. Dubey A, Sung WS, Shaya M, Patwardhan R, Willis B, Smith D, et al. Complications of posterior cranial fossa surgery-an institutional experience of 500 patients. Surg Neurol. 2009;72(4):369-75.

15. Witsch J, Neugebauer H, Zweckberger K, Jüttler E. Primary cerebellar haemorrhage: complications, treatment and outcome. Clin Neurol Neurosurg. 2013;115(7):863-9.

16. Salvati M, Cervoni L, Raco A, Delfini R. Spontaneous cerebellar hemorrhage: clinical remarks on 50 cases. Surg Neurol. 2001;55(3): 156-61 discussion 161.

17. Manno EM, JLD Atkinson, JR Fulgham, EFM Wijdicks. Emerging medical and surgical management strategies in the evaluation and treatment of intracerebral hemorrhage. In: Mayo clinic proceedings, 80(3):420-433. New York: Elsevier; 2005.

18. Elliott J, Smith M. The acute management of intracerebral hemorrhage: a clinical review. Anesth Analg. 2010;110(5): 1419-27.

19. Zahuranec DB, Gonzales NR, Brown DL, Lisabeth LD, Longwell PJ, Eden SV, et al. Presentation of intracerebral haemorrhage in a community. J Neurol Neurosurg Psychiatry. 2006;77(3):340-4.

20. d'Avella D, Servadei F, Scerrati M, Tomei G, Brambilla G, Angileri FF, et al. Traumatic intracerebellar hemorrhage: clinicoradiological analysis of 81 patients. Neurosurgery. 2002;50(1):16-25 discussion 25.

21. Dahdaleh NS, Dlouhy BJ, Viljoen SV, Capuano AW, Kung DK, Torner JC, Hasan DM, Howard MA. Clinical and radiographic predictors of neurological outcome following posterior fossa decompression for spontaneous cerebellar hemorrhage. J Clin Neurosci. 2012;19(9):1236-41.

22. Qureshi AI, Suarez JI, Parekh PD, Bhardwaj A. Prediction and timing of tracheostomy in patients with infratentorial lesions requiring mechanical ventilatory support. Crit Care Med. 2000;28(5):1383-7.

23. Bösel J, Schiller P, Hook Y, Andes M, Neumann J-O, Poli S, Amiri $\mathrm{H}$, et al. Stroke-related early tracheostomy versus prolonged orotracheal intubation in neurocritical care trial (SETPOINT): a randomized pilot trial. Stroke. 2013;44(1):21-8.

24. Navarrete-Navarro P, Rivera-Fernández R, López-Mutuberría MT, Galindo I, Murillo F, Dominguez JM, et al. Outcome prediction in terms of functional disability and mortality at 1 year among ICU-admitted severe stroke patients: a prospective epidemiological study in the south of the European Union (Evascan Project, Andalusia, Spain). Intensive Care Med. 2003;29(8): 1237-44.

25. Jessop ZM, Kane AD, Menon DK. The role of early tracheostomy in patients with posterior fossa haemorrhage in neurocritical care. J Intensive Care Soc. 2012;13(4):293-6.

26. González-García J, Gelabert-González M, García-Allut A, Fernández-Villa JM, López-García E, García-Pravos A. Cerebellar hematomas: a surgically treatable stroke. Rev Neurol. 2000;31(12):1119-26.

27. Tsitsopoulos PP, Tobieson L, Enblad P, Marklund N. Prognostic factors and long-term outcome following surgical treatment of 76 patients with spontaneous cerebellar haematoma. Acta Neurochir (Wien). 2012;154(7):1189-95.

28. Papacocea A, Papacocea $T$, Dănăilă L, Ion $D$, Bădărău $A$, Papacocea R. Primary intracerebellar hematomas: surgical indications, prognosis. Chirurgia (Bucharest, Romania: 1990). 2009;105(6):805-7. 